\title{
Solutions Comparison towards Voice Services Implementation for Operators Starting LTE Deployment
}

\author{
Youness Jouihri, Zouhair Guennoun \\ Laboratoire d' Electronique et de Communications_LEC, Ecole Mohammadia d’ Ingénieurs_EMI, \\ Université Mohammed V-Agdal-UM5A, Rabat, Morocco \\ Email: Jouihri.Youness@gmail.com, zouhair@emi.ac.ma
}

Received January 1, 2012; revised February 3, 2012; accepted March 11, 2012

\begin{abstract}
The continuity of voice services, for many operators planning to start their LTE network, is one of the key requirements to cover back the cost of this expensive network. In this paper, we discuss and compare the possible solutions to add voice services on an LTE network dedicated mainly for high-speed data transfer. By comparing advantages and inconvenient of the existing approaches, we conclude that the selection of the One Voice approach is obvious, especially for operators starting LTE deployments, the One Voice approach has the support of all the famous operators and vendors. Selecting the One Voice approach as a first alternative was evident, but the choice of CSFB as a mid-term solution or backup solution from many operators was not clear. As a key lesson can be learned from the existing technologies, CDMA and GSM, where the type of handset was one of the major factors of the GSM success; we prove in this paper that VOLGA approach is better than CSFB for many reasons: The VOLGA approach uses less complex and less expensive handset while it gives more comfortable user experience compared to CSFB approach, so operators should take into consideration these two points before selecting the second alternative.
\end{abstract}

Keywords: Voice over LTE; One Voice; VOLGA; IMS; SIP; CSFB; SRVCC

\section{Introduction}

LTE is a full IP network designed for data transfer. Moving towards this new technology, mobile operators who receive "over $80 \%$ of their revenues from Voice and SMS" [1], can't ignore the continuity of these two services over data services.

The LTE will be more attractive for operators, if it can replace a hundred years of developments in voice technologies, and provide the same quality or maybe better.

However, until now, voice format is not "defined as a major omission" [1] for the LTE system. Therefore, operators and vendors have to agree on the best solution to transfer the voice services, in this all-IP world.

The remaining sections of this paper are outlined as follows: After an introduction to the SIP protocol, its benefits for the operator and its role to enable the voice over IP services in Section 2. Section 3, explains why IMS is the preferred choice for operators to provide Voice over LTE and its biggest limitation. Section 4 is reserved to present Interim mechanisms used to make the transition to IMS-based call control. Section 5 introduces the VOLGA approach and Section 6 is devoted to the One Voice approach, its advantages and comparison with VOLGA approach. Finally, a summary is provided in Section 7.

\section{SIP Protocol, Evolution, Role and Benefits}

The tendency that all communications are moving to an IP based world was obvious since last few years. The standard to use on this all-IP world was a challenge for mobile operators. SIP protocol was selected and standardized to enable those services related to Voice over IP [2]. Figure 1 is an example where SIP connects the circuit switch to IP Multimedia Subsystem.

For many operators, especially those using MSC Server, the SIP protocol implementation can be very easy, as it is a simple feature working with other MSC Server (MSS) features, and implements the Media Gateway Control Function (MGCF) in the MSS [3]. With the MGCF, the IMS and CS can easily communicate by connecting it to GMSCS (Gateway MSC Server), this gives the networks the CS Interworking functionality with IMS, and so, the VOIP and Video can be forwarded to CS mobile user. The functionality of SIP can be 


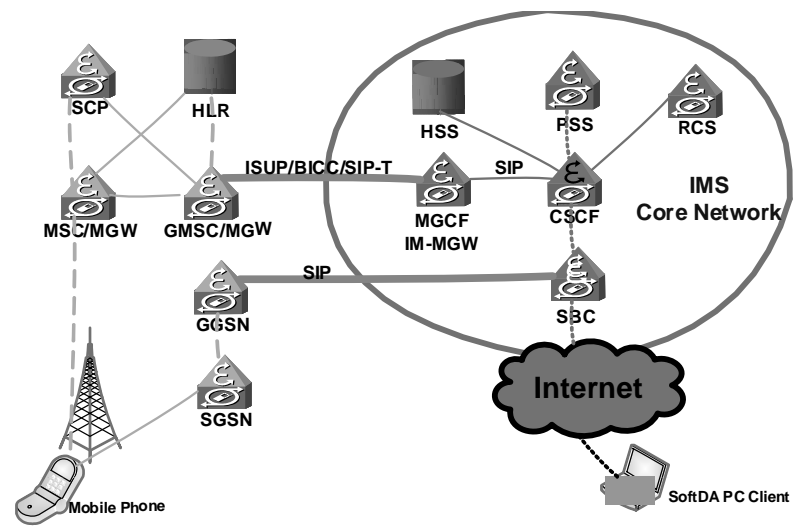

Figure 1. SIP connects the circuit-switched and the IP Multimedia Subsystem domaine (more clarifications about components and interfaces given in [4]).

extended to different interfaces, if we can add the ISUP tunneling facility, for that both SIP-I (Internet Engineering Task Force (IETF)) with ISUP tunneling and SIP-T (International Telecommunications Union (ITU-T) without tunneling should be defined [3].

Towards the IMS, only SIP-T is used without ISUP tunneling. Session refreshment messages are supported on all the interfaces [3]. SIP-T protocol is simply a SIP-I protocol with tunneling OFF [3].

This separation based on ISUP tunneling facility gives a major benefit for this protocol, it can connect the Circuit-Switched (CS) and the IP Multimedia Subsystem (IMS) domains, and provide an open ISUP tunneling SIP trunk (soft switch) interface (SIP-I), that can be used either between the MSSs or towards other equipment's having SIP-I interface, like VMSs (Voice Mail Systems), ATA (Analogue Telephone Adapters), SIP-I gateways and so on.

SIP-I is preferred between MSSs, because it supports GSM or ISDN services more effectively [4]. This feature complies with the 3GPP IMS standards and with the relevant IETF standards. Both the User Datagram Protocol (UDP) and the Transmission Control Protocol (TCP) are supported as signaling transport for SIP. In addition, semi-permanent Stream Control Transmission Protocol (SCTP) associations can be used optionally to transport SIP messages. With SCTP transport, multi-homing can be used as well [4].

\section{IMS Architecture, Evolution and Limitation}

The main focus for Operators starting LTE deployment is the way of delivering their main catch cow "Voice" over LTE, as no clear strategy was defined [1]. Several propositions have been suggested, but IMS was always the favorite choice. With its sophisticated architecture and feature set, developed after more than ten years work by
3GPP, and thousands of pages in different specifications, which in a "way made sure of the continuity of service, interoperability and roaming between operators". Also it can lead the voice evolution story from existing CS networks to a word of IP, after the new convergence to a packet-based access and core network [5]. The IMS can easily connect the Circuit switch and packet switch domain to the applications and services domain, using the facilities given by its main Components shown on Figure 2.

The IMS evolution was very fast, see Figure 3, from one version to the next, more services was provided for operators; but most of the services related to VOIP and CS interworking, subject of this paper was defined on Release 8.

The IMS release 8 was a smooth evolution from previous release building new capabilities:

- IMS centralized services: allow devices using CS connection (GSM/3G CS radio) to use IMS services.

- Multimedia session continuity: improves voice call continuity feature to enable continuity of multimedia media streams when IP access is changed.

- Single-Radio Voice Call Continuity: enables seamless voice handover from LTE (IMS VoIP) to CS.

From the above description we can believe that IMS is the best option for Voice over LTE. IMS release 8 is a complete solution for transferring voice traffic on this all IP word designed for data. With its golden list of features set, we can overcome all the problems and limitations related to voice integration, on a network designed for data.

More than giving smooth continuity of the most important service for operators, IMS is the glue between different domains, so "legacy networks won't go away, IMS supports co-existence with voice infrastructure", and the handover to a $2 \mathrm{G}$ or $3 \mathrm{G}$ circuit switched channel can be done easily once running out of LTE coverage [4].

In parallel IMS support multitude of Application Servers that may give multiple functionality, and realize all the multimedia dreams for operators, but all these facilities make IMS implementation difficult. IMS, unfortunately, is suffering from its own complexity, having a sophisticated but heavy infrastructure, supporting many feature set developed after years of work, the full IMS implementation will cost a lot of time and money for operators, without having any guarantee that end-user will really need and go for all these new services, and so, no insurance to cover back the cost of this big investment. This is why; operators should not go for the IMS bigbong approach.

For Operators starting LTE deployment, the complexity and the multimedia dreams have to be thrown away and they should concentrate on the voice service only, which is the real reason for introducing IMS over their LTE network, "an impossible suggestion only a short 


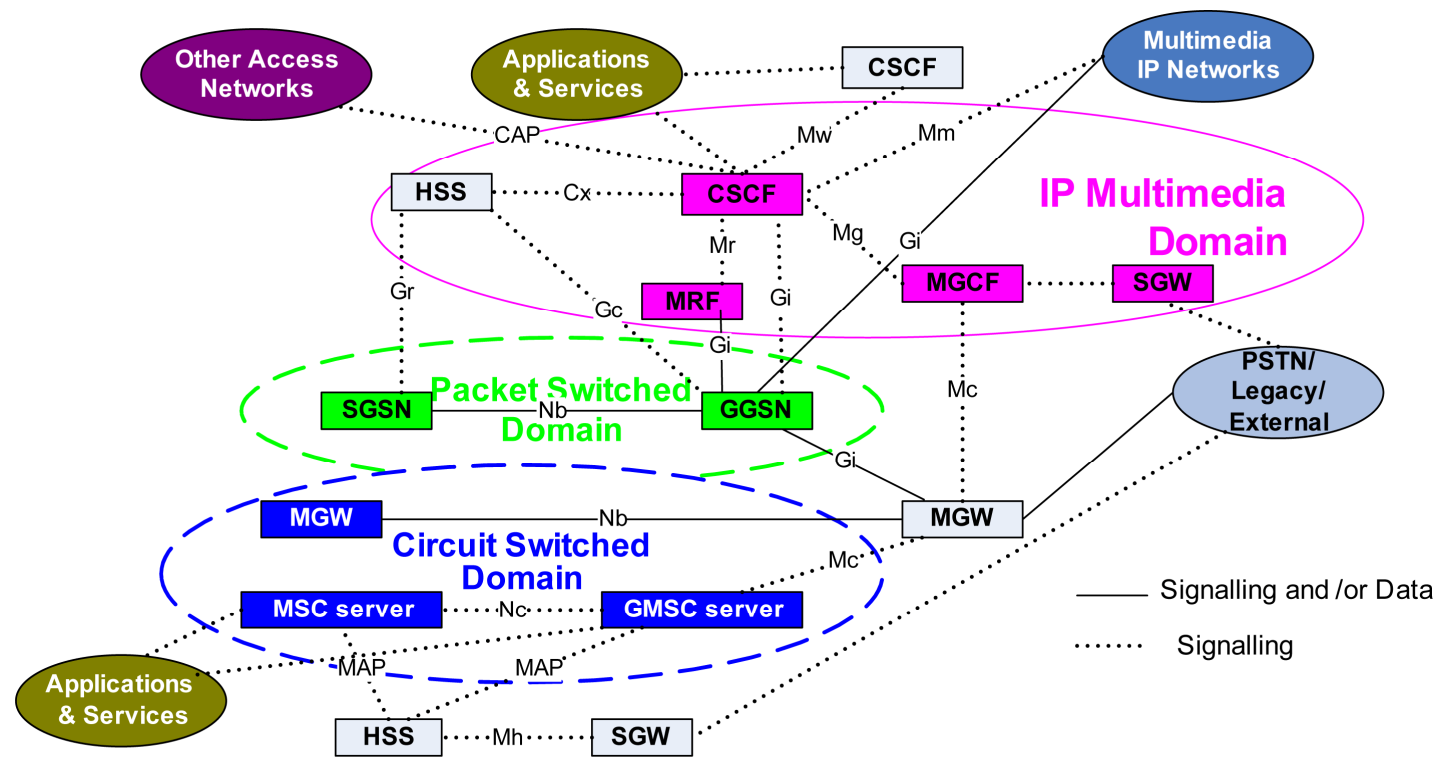

Figure 2. IMS detailed architecture (Release 5) (Interfaces description and components given in [4]).

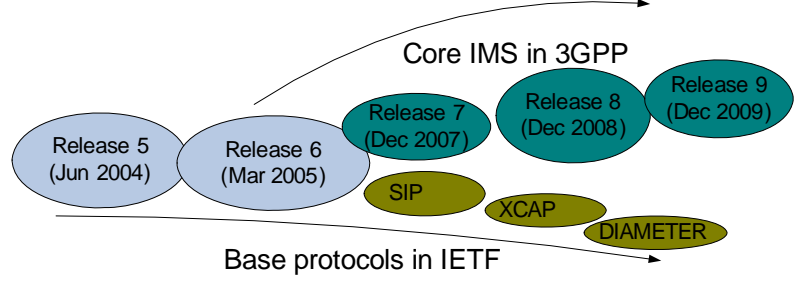

Figure 3. Evolution of IMS in relationship with SIP protocol [4].

time ago for all who select IMS approach, but the pressure seems to be mounting” [6], they cannot concentrate on implementing a full IMS network and leave their main objective for creating a real network IP based. The first focus should be on voice, make it working, instead of investing heavily in building the infrastructure and waiting for the subscribers and services to come, after that they can target the services that subscribers are likely to embrace then, building up the infrastructure to support those services incrementally, by adding the IMS-peripheral components needed, also check the possibility to reuse smartly their existing network, by using facilities that can change their components to IMS component as shown in Session 2, where operators using MSC servers can implement the Media Gateway Control Function (MGCF) in their MSS by adding SIP feature. The replacement of the entire functional systems will not be required, till reaching a point where the sophistication of the services and the operational saving of IMS will become obvious; after that the adoption will accelerate toward a full IMS network.

The IMS pragmatic approach is needed as the full implementation can take several years. So, which Interim mechanisms can be selected to make the transition happen?

\section{Interim Mechanisms for IMS-Based Call Control Transition}

Today, all the parties agreed, 3GPP and GSMA, that IMS will become the mainstream choice for voice over LTE, and will be the standard architecture for mobile voice [7], but as explained above, operators are not yet ready to adopt this approach, even more, handset makers has to create devices that support 3GPP Release 8 and Release 9 functions that work with a VOLTE network. To give more time for network and handset to be ready to support IMS big-bong approach, the use of interim mechanisms became mandatory. Many approaches already exist, and the best selection will give definitively a smooth IMS deployment. So let us describe these approaches before making any selection.

The first approach, circuit switched fallback (CSFB), where LTE will provide just data services, and for the initiation or the reception of a voice call, fallback to CS domain will be performed [7].

Figure 4 illustrates the case, when a subscriber wishes to make a voice call, in a CS Fallback to legacy 3GPP network architecture, the UE makes a service request to the LTE network, and the coordination with the 3GPP network will redirect the UE to the legacy CS network. For mobile terminated calls, the subscriber is paged in the LTE network and he cannot move to CS network till subscriber decides to accept the call [8]. For SMS, it can be sent without leaving the LTE network (the handset can use interface known as SGs which allows messages to be sent over an LTE channel [1]).

For many operators, they need just to upgrade their MSC to support CSFB [7].

However, CSFB may cause LTE data connection drop 


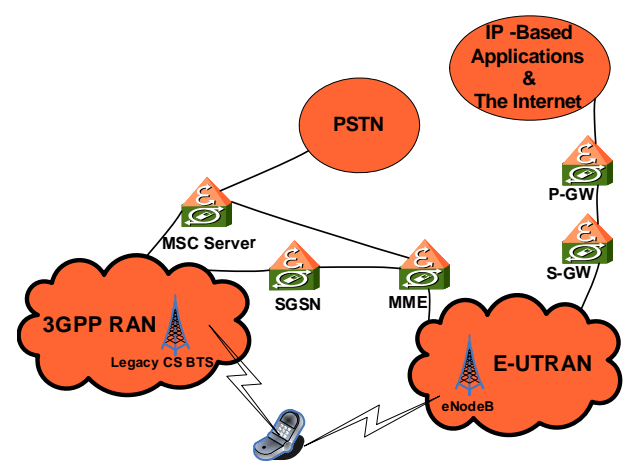

Figure 4. Circuit switched fallback architecture [8].

and entail an additional call set up delay [7]. The CSFB might not be the best interim solution, but one of the most important addition that we can get by adopting it, is the scenario of LTE roaming [7]. "For example, when the visited network does not have the IMS or when the IMS roaming protocol is yet to be deployed, CSFB can provide voice-call service for inbound LTE roamers" [7].

The second approach, dual radio handset approach or simultaneous voice and LTE (SVLTE), is "solely based on the handset", no changes are required on the network and the IMS deployment is either not needed, the handset will simultaneously get the data services from the LTE and the voice service from CS mode. This solution can suffer from the fact that "the phone can become expensive with high power consumption" [7].

The third approach is the approach founded on the usage of over-the-top (OTT) where applications like Skype and Google Talk can provide LTE voice service. The LTE "can create natural convenience for the development of OTT voice calls" and break almost all the barriers, by using its features like broad bandwidth, low latency, being always-online, and All-IP [7].

This approach cannot receive much support, as no operator can handover completely its main driver of revenue, voice, to the OTT actors.

For the fourth and fifth approaches, VOLGA and One Voice are the most applicable approaches to meet the consumers' expectations, according to their impressive list of supporters. Very big debate and polemics were initiated on different forums to decide which approach will be the final solution to exceed the voice challenge on LTE network. Next two chapters will be reserved for these two approaches since more details and clarifications are needed to reach a verdict about the best option to be adopted.

\section{The VOLGA (Voice over LTE via Generic Access) Approach}

The VOLGA approach was initiated during the VOLGA Forum on March, 2009 for extending traditional voice and SMS services over LTE access networks, without requiring a re-architecture of the network, one of the very low cost methods of getting the two highest drivers of revenue services for operators [1]. The concept is very simple, instead of creating the core telephony services in IMS, we simply make the existing telephony infrastructure a packet service delivered over IP via LTE, which means making use of GSM signaling over IP, and elevate the core voice network to be able to deliver packet service over the LTE access network. The main idea was to adapt Universal Mobile Access/Generic Access Network (UMA/GAN) system to LTE; "Whether through Wi-Fi radios (Nanoradio, Swedish Wi-Fi radio start-up) or fixedline services (T-Mobile's highly successful fixed-line service), it has been proven that the UMA/GAN is a very successful platform to deliver mobile voice (and data) services over IP access networks” [9], so we should also check it on the LTE networks.

The aim of VOLGA is to make use of the existing mobile core, which is extremely feature rich, to deliver the primary revenue generating service for operator, by "tunneling circuit switched voice traffic across LTE from the generic access network controller (GANC) server" to the evolved packet core in LTE [9], as shown in Figure 5. For handover from LTE to 2G/3G CS, the feature Single Radio Voice Call Continuity (SRVCC) can be used [10]. The use of this feature contributes a lot to make VOLGA better option, in comparison to the previous interim approaches, especially CSFB. In addition of enabling the full range of the current voice services and made them available, contrarily to the services given by CSFB, the SRVCC gives subscribers chance to use a single radio mode device, which is less complex and less expensive, also the switching to CS network will be needed only when terminal roams out of LTE coverage, so the call setup time will be less, since time is required only out of LTE [11]. On the other side CSFB needs a dual mode device, complex and expensive due to the increase of network signaling load, also the switching to CS network is needed for every mobile originating or terminating call, which augment the voice call setup [11].

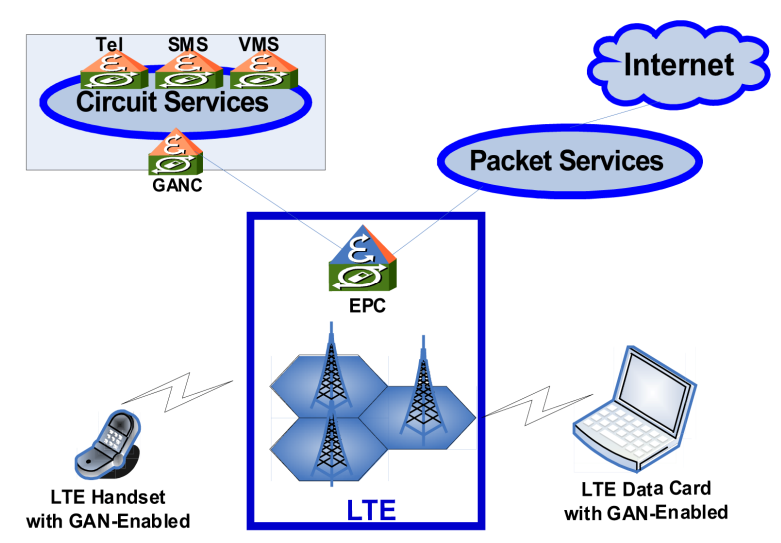

Figure 5. Elevate existing core voice services to become packet services over LTE [9]. 
So from the above description, VOLGA beat CSFB with the various advantages mentioned previously, we can even add more, like the protections of the existing and expansive voice core investment, the smart exploitation of an existing standard GAN already "proven as an effective and robust method for extending mobile voice services over broadband packet access networks" [9], in addition VOLGA is supported by all major handset vendors and it can clear all the confusion for an early handset development [9]. This confusion can be cleared by "defining the LTE voice to be identical to the existing voice services of the 3G and 2G networks" [9], so LTE will not face the same problem of the 3G's deployment where "operators waited years for viable handsets" [9].

The VOLGA can also win the battle hands down against the dual radio handset approach by referring to the type of handset that can be used by the last approach, expensive and should support the high power consumption. A key lesson can be learned from the existing technologies, CDMA and GSM. Many things makes GSM more famous than CMDA, but I think, the major factor of success was the handset, GSM gave more flexibilities (change the phone, buy new versions) and choices to the end user than CDMA.

For the OTT approach, there is no need to go through details, and make technical comparison between this approach and the VOLGA. Logically no operator can give the best source of revenue to OTT actors, so the VOLGA selection will be obvious, since it will keep their resource save.

The VOLGA can be considered as a mid-term solution for operators preferring the migration of all services to IMS; as we proved previously that this approach has more chance than all previous approaches, to replace IMS for telephony services delivery [9]. The VOLGA can also be considered as a long term solution for operators willing to invest in LTE for non-voice services, and keep their existing 2G/3G network for the telephony services [9]. The handset and the telephony services must support 2G/3G as a fall back even after full LTE deployment, this can guaranty the use of VOLGA as long term proposition [9]. The VOLGA solution is not yet standardized by 3GPP, many operators and vendors were supporting this approach, but now they join the group adopting the new approach called One Voice [12]. So what is this approach? And how it took the support of many major vendors from VOLGA?

\section{The One Voice Approach: Its Advantages and Its Comparison with VOLGA Approach}

The One Voice approach was announced on November 2009 after coordination between "six operators, three core networks vendors and three handsets manufacturers". The idea was to use the concept of IMS MMTel feature, already defined by 3GPP in IMS release 6, "to a minimum set of functionality for a voice service equivalent to what we have today in CS domain" [13]. After the adoption of this approach by GSMA on January 2010, One Voice started to attract different parties, especially when it was announced publicly on February 2010 at MWC (mobile word congress) [13]. GSMA has added three key interfaces, to address the entire end-to-end voice and SMS ecosystem, by also focusing on Roaming and Interconnect interfaces, in addition to the interface between customer and network [13].

The First interface, UNI (User Network Interface), as shown in Figure 6, is located between the user's and the operator's network. Define a common User Network Interface enables the benefice of maintaining roaming capability, giving Global scale and global ecosystem and greater amortization of $R \& D$ as a result of lower cost per unit for vendors [14].

The second interface, I-NNI (Interconnect Network Network Interface), as shown in Figure 6, is the interface located between the networks of two parties making a call, creating an end-to-end call connectivity, which required a common functionality and a common implementation to remove the need of expensive interworking functionality [14].

The third interface, Roaming Network Network Interface is an interface located between the home and visited network, used by users roaming in visited networks, not attached to their home network. It is the most challenging interface, as it required compatibility between different networks, and the incompatibility with any roaming solution of other networks, might potentially affect the voice service of the roamers on these networks [14].

The biggest challenge was the definition of a functional split between Home and Visited networks, as the 3GPP has different options for split [15], so the One Voice approach will select one. The selection will be based on the minimum requirements of this approach, means having the voice service equivalent to what we have today in CS domain.

The Figure 7 shows the three roaming options given by 3GPP: The first alternative has the ability to meet the regulatory requirements for roamers in the visited network, such as Lawful intercept and Emergency call by using the VOIP emergency calls via visited network, also the LI is possible on the visited network [15].

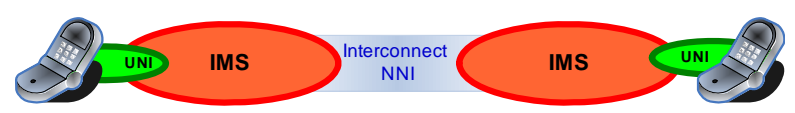

Figure 6. User Network Interface (UNI) and Interconnect Network Network Interface (I-NNI) [14]. 


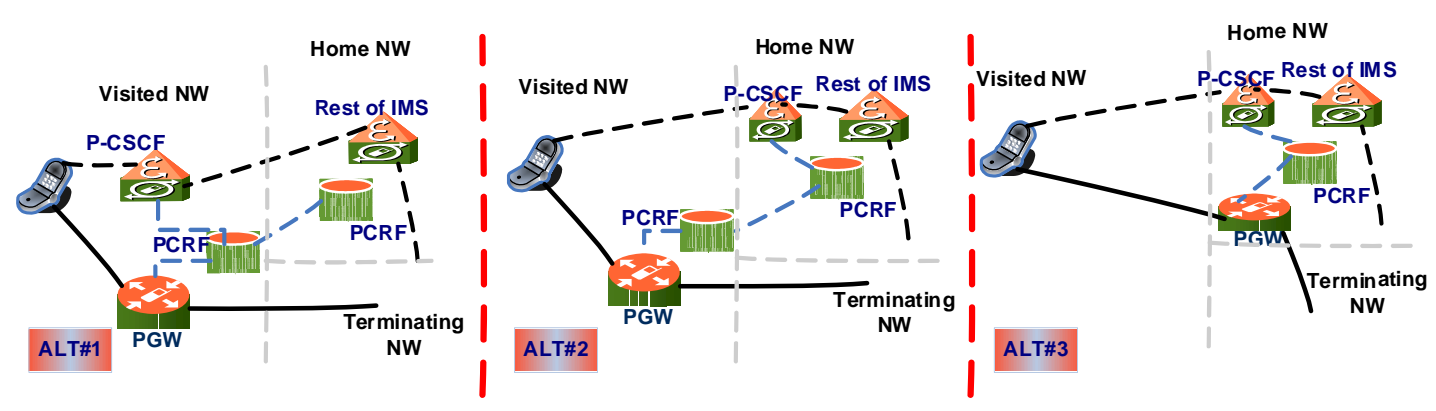

Figure 7. 3GPP architecture contains three roaming options [15].

The two other alternatives cannot give this ability; as for emergency call, the VOIP requires access via visited P-CSCF or emergency call via CS domain, and for Lawful interception, the Control plane interception is not possible if IPSec applied.

Obviously, alternative one will be selected, as it can provide the needed requirement. The functional split selected by One Voice makes the user experience more comfortable [15], as it gives the ability to optimize the media path for voice sessions, and minimize the number of media gateways and transcoding for user plan, which reduce the call setup time. After this selection we have a common roaming network interface which allow interoperator communication, policy control and enforcement, and inter operator charging.

The Work of GSMA will not be limited on the addition of new interfaces, but it will encompass the Key technical requirements to make a nice voice experience on a network having different technologies. For an operator starting LTE deployment, the LTE coverage will be very limit and handover to legacy networks will be a big requirement. So it will be necessary to ensure the continuity of the voice calls when user moves from LTE to legacy networks, this handover can be achieved using Single Radio Voice Call Continuity (SRVCC) [11]. The SRVCC is an optional feature included in One Voice specification, allows devices with one radio access technology at a time with an ongoing voice call, to transition to the circuit-switch domain in the event of loss of LTE coverage, this feature will significantly increase the complexity of the network as PS and CS domains need to support it [11], but for operators on the starting phase of LTE deployment, the selection of SRVCC will make their network more comfortable for users, because without it, they will suffer from the high number of calls drop related to the loss of LTE coverage. The complete handover procedure is given in [11]. For the SMS part, which is a CS service that needs special attention with regard to LTE, the Target SMS solution will be based on IMS when the UE and network may support the SMS-over-IP, the migratory solution will be SMS over SGs as defined in 3GPP [16].
As described before, the One Voice approach is an IP Multimedia Subsystem used at its minimum capacity to provide Voice over LTE, so the One Voice approach is definitively the best solution to reach the target solution IMS, and realize the multimedia dream of many operators, as we can provide any new service, that subscribers are likely to embrace, by adding the needed IMS-peripheral components, without replacing the entire functional systems, progressively we will reach the full IMS configuration. The VOLGA approach will use different system, so the pragmatic IMS approach cannot be adopted on it.

Today, the OTT actor's services became more sophisticated and less expensive, especially for international calls, so many users start using it in state of using expensive legacy network, and so, operators start losing some of their permanent source of revenue [17]. Providing the same voice experience as legacy network cannot be a permanent solution, as the competition is increasing day by day, and customers start adopting the new broadband wireless technology, so they will not accept lower quality voice service with higher price, operators should either give a very good billing discount which will affect their revenue, or either provide an HD (high definition) voice services, which enhances voice quality using a wider frequency range, so mobile operators should be able to offer nice voice quality, even higher than landline voice, like that they can compete the OTT actors and save their revenue.

VOLGA cannot give a voice quality better than the existing network, as the approach is based on the existing system, so the HD option is not possible on this approach.

Finally, the list of supporter of One Voice is still increasing, and the major operators and vendors are already there, some of them drop their support to VOLGA and join the One Voice group [12]. Having a big agreement for a specific technology makes life easy for operators especially in case of roaming, so the technologies collecting more support will be obviously the successful one and the best choice for any operator on the starting phase of deployment. 


\section{Summary}

This paper has analyzed the different solutions to implement the voice services over LTE network. After comparing these approaches, we have presented for operators starting their LTE network, the best selections to support the voice services over LTE. Our results match with the actual tendency, where all the major operators and vendors went for the One Voice approach as a primary alternative. But as a mid-term or secondary alternative, we proposed the VOLGA approach contrarily to a big number of operators who want to go for CSFB. As Yankee Group Research Director Brian Partridge said referring to VOLGA approach, “The reason you haven't seen a lot of operators is because they haven't studied it yet” [18]. On this paper we conclude, after studying all aspects, that we should not throw away the VOLGA approach. We proved that VOLGA is better than CSFB for many reasons. So, operators planning to start this big investment should go through all the details of VOLGA approach. As a wrong selection can badly affect the evolution of a new technology, and the history of mobile technologies have already proved it.

\section{Acknowledgements}

The authors would like to acknowledge Mr. Faisal M. J. Attieh for his valuable advice and support of this work.

\section{REFERENCES}

[1] Cellular Telecoms, "Voice over LTE-VoLTE,” 2011. http://www.radio-electronics.com/info/cellulartelecomms/ lte-long-term-evolution/voice-over-lte-volte.php

[2] K. Banerjee, "SIP (Session Initiation Protocol) Introduction,” 2009. www.siptutorial.net/SIP/index.html

[3] Nokia Siemens Networks, "Feature 1331: Session Initiation Protocol in the MSC Server,” 2009.

[4] A. Bencheikh, "Introduction to IMS \& SDP," Al Akhawayn University, Ifrane, 2010.

[5] AT \& T, et al., “One Voice: Voice over IMS Profile,” 2009. www.nokiasiemensnetworks.com/system/files/document/ One_Voice_Profile_0.pdf

[6] M. Sauter, "The IMS One Voice Profile-Some Thoughts," 2009.

http://mobilesociety.typepad.com/mobile_life/2009/11/th e-ims-one-voice-profile-some-thoughts.html

[7] Q. H. Chen, "Evolution and Deployment of VoLTE," 2011. www.huawei.com/en/static/hw-094164.pdf

[8] I. Tanaka, T. Koshimizu and K. Nishida, "CS Fallback Function for Combined LTE and 3G Circuit Switched Services," NTT DOCOMO Technical Journal, Vol. 11, No. 3, 2009, pp. 13-19.

[9] S. Shaw, "LTE: Delivering Telephony Services in an LTE Network," 2009.

www.smart-wi-fi.com/pdf/uma_mag_spring_09_lo.pdf

[10] Ericsson White Paper, "Voice over LTE,” 2010. www.ericsson.com/res/docs/whitepapers/voice-over-lte.p df

[11] S. Vittal, "SRVCC: Single Radio Voice Call Continuity with LTE,” 2011. www.slideshare.net/allabout4g/wpsrvccwithltepdf

[12] M. Donegan, "MWC 2011: Deutsche Telekom Ditches VoLGA," 2011. www.lightreading.com/document.asp?doc_id=204581

[13] D. Warren, “Voice \& SMS over LTE Update,” 2011. https://infocentre.gsm.org

[14] D. Warren, "Voice over LTE,” 2010. https://infocentre.gsm.org

[15] Nokia Siemens Networks, "GSMA LTE Voice Roaming,” 2010. https://infocentre.gsm.org

[16] D. Warren, “Next Steps for VoLTE,” 2010. https://infocentre.gsm.org

[17] Juniper Networks, “The Evolution of Business Services: Delivering Value to Boost Revenue,” 2011. www.juniper.net/us/en/local/pdf/whitepapers/2000305-en .pdf

[18] S. Shaw, "Let's Give 'Em Something to Talk About," 2011.

http://voiceoverlte.typepad.com/voice-and-sms-over-lte/2 009/10/lets-give-em-something-to-talk-about.html 